\title{
A Wavelet Based Solar Radiation Prediction in Nigeria Using Adaptive Neuro-Fuzzy Approach
}

\author{
Sani Salisu ${ }^{1}$, Mohd Wazir Mustafa ${ }^{2}$, Mamunu Mustapha ${ }^{3}$ \\ ${ }^{1,2,3}$ Faculty of Electrical Engineering, Universiti Teknologi Malaysia, Johor Bahru, 81310 Johor, Malaysia \\ ${ }^{1}$ Department of Electrical Engineering, Ahmadu Bello University, Zaria, Nigeria
}

\begin{abstract}
Article Info
Article history:

Received Jan 21, 2018

Revised Apr 15, 2018

Accepted Nov 4, 2018

Keywords:

ANFIS

Wavelet Transform

Solar radiation

Meteorological data

Nigeria

ABSTRACT

In this study, a hybrid approach combining an Adaptive Neuro-Fuzzy Inference System (ANFIS) and Wavelet Transform (WT) is examined for solar radiation prediction in Nigeria. Meteorological data obtained from NIMET Nigeria comprising of monthly mean minimum temperature, maximum temperature, relative humidity and sunshine hours were used as inputs to the model and monthly mean solar radiation was used as the model output. The data used was divided into two for training and testing, with $70 \%$ used during the training phase and $30 \%$ during the testing phase. The hybrid model performance is assessed using three statistical evaluators, Mean Absolute Percentage Error (MAPE), Root Mean Square Error (RMSE) and Coefficient of determination $\left(\mathrm{R}^{2}\right)$. According to the results obtained, a very accurate prediction was achieved by the WT- ANFIS model by improving the value of $\left(\mathrm{R}^{2}\right)$ by at least $14 \%$ and RMSE by at least $78 \%$ when compared with other existing models. And a MAPE of $2 \%$ is recorded using the proposed approach. The obtained results prove the developed WT-ANFIS model as an efficient tool for solar radiation prediction.
\end{abstract}

Copyright $\odot 2018$ Institute of Advanced Engineering and Science. All rights reserved.

\section{Corresponding Author:}

Sani Salisu,

Faculty of Electrical Engineering,

Universiti Teknologi Malaysia,

Johor Bahru, 81310 Johor, Malaysia.

Email: s.salisu@live.com

\section{INTRODUCTION}

Over the years, there have been a lot of interests in the use of renewable energy as an alternative energy source due its availability in abundant quantity. The inexhaustible nature of this energy sources couple with the fact that its clean, free and also environmentally friendly makes it an alternative source of energy across the globe [1]. Several renewable energy sources have been utilized for energy generation across the globe with solar energy being the most exploited energy source. Solar energy is readily and abundantly available in Nigeria. The availability of solar energy in Nigeria makes it possible to have a successful solar power project [2]. Solar power design is only achievable with accurate knowledge of solar radiation of the project area [3]. This is required so as to have an effective and efficient solar power design that will be able to supply the required load demand and also have proper knowledge of the energy that can be generated from the project area.

Specific equipment are designed for recording horizontal solar radiation but are not readily available in Nigeria due to some reasons. High cost of the equipment coupled with high maintenance cost led to nonpatronage of the equipment by some developing countries [4]. The unavailability of these equipment in many meteorological stations led to the development of different empirical and artificial intelligence models for solar radiation estimation using the available meteorological data that have strong correlation with solar radiation [5]. In Nigeria, all the 36 states have a government owned meteorological agencies for recording 
meteorological data but none of these states has a single equipment for recording solar radiation. Due to this reason it is important to develop a model to predict the horizontal solar radiation for Kano, Nigeria.

Several researches have been conducted and several models have been developed in various parts of the world to predict horizontal solar radiation. Researchers have conducted these investigations using the available meteorological data, [6-9] which include minimum temperature, sunshine hours and maximum temperature using different approaches. In places where the records of the solar radiation data were not available, [10-12] utilized the available data to develop the temperature based models for horizontal solar radiation prediction using minimum temperature, average temperature and maximum temperature. The estimation was also found to be accurate and efficient.

In this study, a hybrid WT-ANFIS approach is investigated for horizontal solar radiation prediction in Nigeria using the available meteorological data. Adaptive Neuro-Fuzzy Inference System (ANFIS) coupled with wavelet transform (WT) are utilized for solar radiation prediction in Nigeria. Monthly mean sunshine hours, minimum temperature, maximum temperature and relative humidity were used as the input while solar radiation is used as the output. ANFIS is used to train and test data for the prediction while the WT is used before the prediction to clean the data. ANFIS is a strong and regularly used hybrid logical system which combines the representation of fuzzy logic and the learning rule of neural network. ANFIS has been extensively exploited by several researchers [5], [13-16], for horizontal solar radiation and other engineering applications. WT is a signal processing tool used in decomposing and reconstructing signals or data into different frequency components [20]. The main objective of this study is to propose a new WTANFIS approach and investigate its efficiency and accuracy for horizontal solar radiation prediction in Nigeria. The obtained results are compared with ANFIS model and other existing models [5], [11], [12], [15] and [17] for validation.

\section{RESEARCH METHOD}

\subsection{Study Location}

In this study, the meteorological data used was recorded at Nigerian meteorological agency (NIMET) Kano, Nigeria, with longitude $12.0022^{\circ} \mathrm{N}$ and latitude $8.952^{\circ} \mathrm{E}$ [18]. 10 years data ranging from (2002-2012) were for the ANFIS-WT model training and testing. The meteorological data used for this study has strong correlation with horizontal solar radiation, which includes minimum temperature, maximum temperature, relative humidity and sunshine hours. The horizontal solar radiation for Kano at latitude $12.0022^{\circ} \mathrm{N}$ and longitude $8.952^{\circ} \mathrm{E}$ was obtained from National aeronautics and space administration NASA [9]. The data was divided to two for both the training phase and testing phase. $70 \%$ of the data were used for training and $30 \%$ were used for testing.

\subsection{Wavelet Transform}

Wavelet transform is a signal processing tool used in decomposing signals or data into different frequency components. It has a wide application in Engineering and scientific applications [20, 21] especially where data and signal analysis are required. Wavelet is used to decompose time series signal into approximate and detail components to reduce the variation between the data series [22, 23] In WT results of the analysis are reconstructed for further analysis using inverse-WT. Depending on the application, the decomposition and reconstruction is in to levels, and based on selection of an appropriate mother wavelet, illustrated in Figure 1.
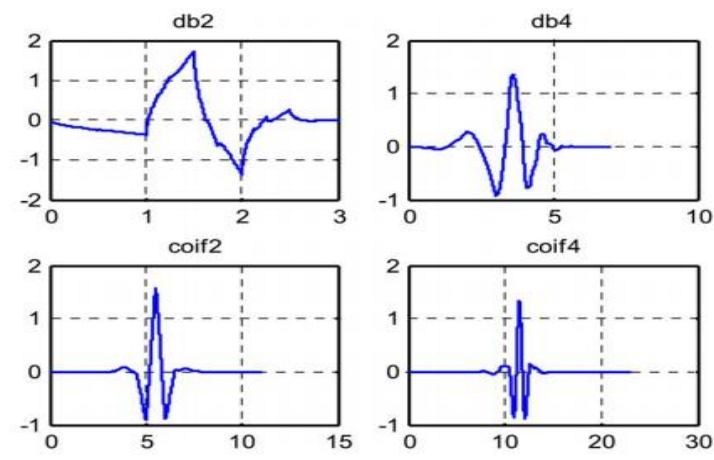

Figure 1. Example of four Mother wavelets functions 
The decomposition and reconstruction process is shown in Figure 2. Equation (1) illustrates a decomposed signal, $S(t)$ using Equation (2).

$$
S(t)=A_{n}(t)+D_{n}(t)+D_{n-1}(t)+D_{n-2}(t)+\ldots \ldots \ldots
$$

where $A_{n}(t)$ is the approximate component and $D_{n}(t), D_{n-1}(t), D_{n-2}(t)$, etc, are detail components.

$$
W T_{(a, b)}=\frac{1}{\sqrt{a}} \int_{-\infty}^{\infty} s(t) \psi\left(\frac{t-b}{a}\right) d t
$$

Where $\psi(t)$ is the mother wavelet, $a$ is scale factor and $b$ is the time-shift parameter. The reconstruction of the data is conducted using Equation (3) with the all parameters maintaining their original definitions.

$$
s(t)=\frac{1}{c_{\psi}^{2}} \int_{a} \int_{b} \psi_{\psi}(a, b) \frac{1}{a^{2}} \psi\left(\frac{t-b}{a}\right) d a d b
$$

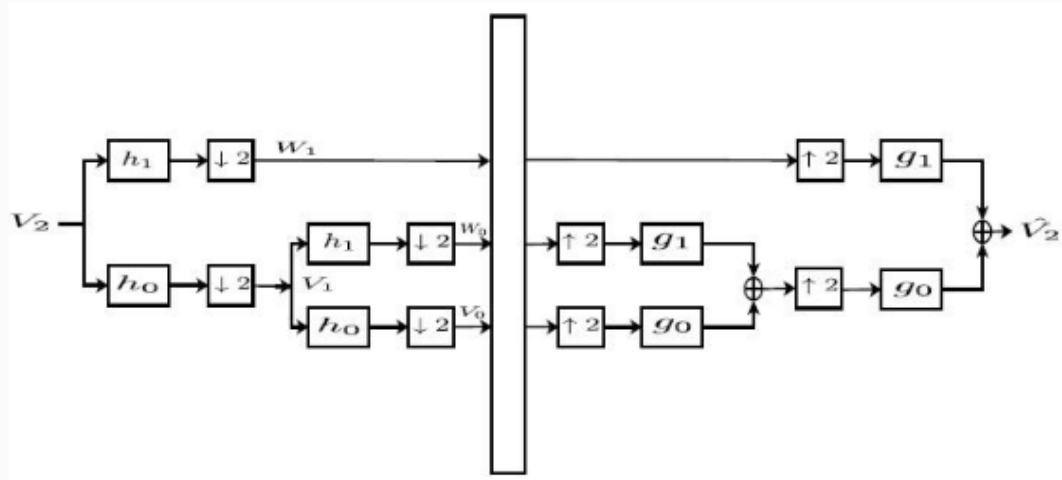

Figure 2. Two levels wavelet decomposition and reconstruction diagrams

\subsection{Adaptive Neuro-Fuzzy Inference System}

ANFIS was first developed by J.S Roger in the year 1993 by combining fuzzy logic system and neural network [24]. The ANFIS is a form of neural network that functions like the Sugueno-type "IF....THEN" fuzzy inference system rule being a network structure and is considered to be more efficient than the individual neural network or fuzzy logic system, it provides more optimal solution than any of the two system [25]. A typical ANFIS structure is presented in Figure 3 with two inputs $x$ and $y$ and one output $f$, it also consist of five layers with each layer having different function. The ANFIS used for this study comprises of four inputs and a single output. Each of the five layers consist of nodes, the nodes on each layer perform the same functions.

$$
\begin{aligned}
& \text { 1. If } \mathrm{x} \text { is } \mathrm{A}_{1} \text { and } \mathrm{y} \text { is } \mathrm{B}_{1} \text {, then } f_{1}=p_{1} x+q_{1} y+r_{1} \\
& \text { 2. If } \mathrm{x} \text { is } \mathrm{A}_{2} \text { and } \mathrm{y} \text { is } \mathrm{B}_{2} \text {, then } f_{2}=p_{2} x+q_{2} y+r_{2}
\end{aligned}
$$

where $p_{i}, q_{i}$ and $r_{i}$ are subsequent parameters. 


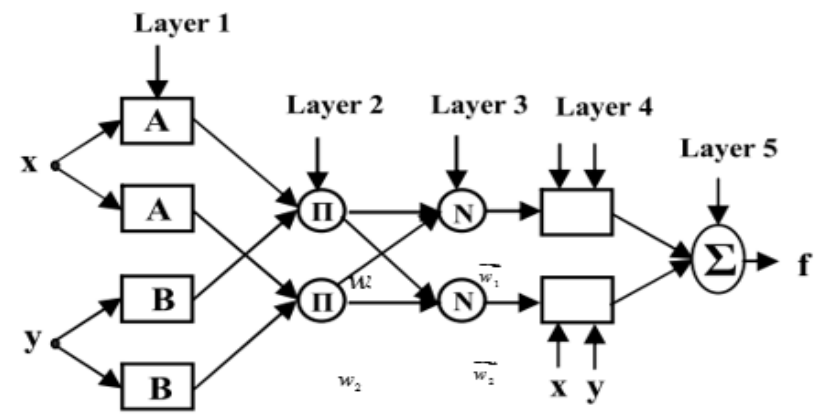

Figure 3. A typical ANFIS structure

Layer 1: The first layer consist of the input membership functions and supplies them to layer two. Each node in this layer has a node function and is also an adaptive node. The nodes output are presented in Equations 6 and 7.

$$
o_{j, i}=\mu_{A_{i}}\left(x_{i}\right) \text { for } i=1,2
$$

Or

$$
o_{j, i}=\mu_{B_{i-1}}\left(y_{i}\right) \text { for } i=3,4
$$

$\mu_{A_{i}}\left(x_{i}\right)$ and $\mu_{B_{i-1}}\left(y_{i}\right)$, denotes the membership functions of the node A, while node $i$ comprises of $x$ or $y$ as its input, and $A_{i}$ or $B_{i-1}$ is a connected verbal label. $O_{j, i}$ is the membership score of sets A and B fuzzy. The global function of the non-linear constraints is presented in Equation (8) [26] and [27].

$$
\mu_{A_{i}}\left(x_{i}\right)=\frac{1}{1+\left(\frac{x-c_{i}}{a_{i}}\right)^{2 b_{i}}}
$$

where $a_{i}, b_{i}, c_{i}$ are the sets of variable. This function varies as the values of the variable changes, hence exhibiting diverse membership functions type for set A fuzzy.

Layer 2: The signals coming from the first layer are multiplied and the results are sent out as the output of this layer. The output is deliberated as an AND or OR procedure of the membership function that comes from preceding layer [28]. It is presented in Equation (9).

$$
o_{i, 2}=w_{i}=\mu_{A_{i}} \times \mu_{B_{i}} \times \mu_{C_{i}} \ldots \ldots
$$

Where $\mu_{A i}$ denotes the membership function of node A and $\mu_{B i}$ is the membership function of node $\mathrm{B}$

Layer 3: Layer three is called the normalization layer and a non-adaptive layer, this layer usually make the rules. The ratio of the node's firing strength to the sum of all the firing strengths going into the node. This layer is non-adaptive layer [29].

$$
o_{i, 3}=\overline{w_{i}}=\frac{w_{i}}{w_{1}+w_{2}+\ldots \ldots}
$$

$w_{i}$ represents the firing strengths. 
Layer 4: all the nodes in layer four are adaptive nodes with node function. This means the product of the signal controlled from the preceding node gives node $i[29,30]$.

$$
o_{i, 4}=\bar{w}_{i} f_{i}=\bar{w}_{i}\left(p_{i} x+q_{i} y+r_{i}\right)
$$

where $\overline{w_{l}}$ is the normalized firing strength of node $i$ from layer three and $p_{1}, q_{1}$ and $r_{1}$ are the subsequent parameters

Layer 5: this is the last layer of the ANFIS structure that consist a single node, this node is non-adaptive and also called a fixed node. It is the summation of all signals coming from the preceding layer and compute them as the total output [24], [28].

$$
o_{i, 5}=\sum_{i} \bar{w}_{i} f_{i}
$$

where $f_{i}$ is summation of the subsequent parameters of the fourth layer.

\subsection{Model statistical evaluators}

The ANFIS-WT model performance is evaluated using the statistical evaluators in Equations (13-15)

1. Root mean square error (RMSE)

$$
R M S E=\sqrt{\frac{\sum_{i=1}^{n}\left(o_{i}-\bar{P}_{l}\right)^{2}}{n}}
$$

2. Mean absolute percentage error (MAPE)

$$
M A P E=\frac{100}{n} \sum_{i=1}^{n} \frac{\left|Y_{i}-\widehat{l}_{l}\right|}{\left|Y_{i}\right|}
$$

3. Coefficient of determination $\left(R^{2}\right)$

$$
R^{2}=\frac{\sum_{i=1}^{n}\left(O_{i}-\overline{O_{l}}\right)^{2} \cdot\left(P_{i}-\overline{P_{l}}\right)}{\sum_{i=1}^{n}\left(O_{i}-\overline{O_{l}}\right) \cdot \sum_{i=1}^{n}\left(P_{i}-\bar{P}_{l}\right)}
$$

where $O_{i}$ and $P_{i}$ are the estimated and experimental values, and $\bar{O}_{i}$ and $\bar{P}_{i}$ are the mean values of $O_{i}$ and $P_{i}$. Also, $\mathrm{n}$ represents the entire amount of test data. Higher values of $R^{2}$ indicates good model performance while lower values of RMSE and MAPE also show good performance.

\subsection{Model Development}

This section describes the procedure for the model development as presented in Figure 4 . WT is applied on the time series data used for the ANFIS prediction. The data gotten is first decomposed into two levels of wavelet coefficients using Db2, and later reconstructed as shown in Figure 2. The estimated signals from the WT-ANFIS model form the estimated output of horizontal solar radiation.

Four input parameters, relative humidity, sunshine hours, maximum temperature, minimum temperature and one output horizontal solar radiations were used to train and test the ANFIS-WT model. A total of 120 sets of data over twelve years were used. The data sets were divided into two sets for both training and testing phases. $70 \%$ of the data ranging from 2002-2008 were utilised during the training phase and $30 \%$ ranging from 2009-2012 utilised during the testing phase.

The data used is first presented on an excel sheet in a matrix form with first four columns representing the input data and the last column representing the output data. These columns representing the input data are represented as the real inputs. Before the estimation, wavelet transform is used to decompose the data at two levels using $\mathrm{db} 2$ mother wavelet. The $\mathrm{db} 2$ is chosen because it is widely agreed that it can give good approximation on the signals [22]. From the decomposed signals, two details and approximate coefficients are selected because they give more information on the used data. Since we have three sets of coefficient signals, three different ANFIS networks are needed to train each coefficient. The next step is to forward the wanted signals from WT to the ANFIS structure. The WT data is then used to train the ANFIS, the parameters of the ANFIS are adjusted during the training phase to satisfy the submitted outputs. The same procedure occurs during the testing phase using the data that was not used at the training phase. 
The outputs of the ANFIS are further extracted and reconstructed using the WT. The reconstructed outputs give the final output of the horizontal solar radiation prediction by WT-ANFIS approach. This is followed by computing the error margin between the predicted output and the targeted output. The performance of the WT-ANFIS model is evaluated using $\mathrm{R}^{2}$, MAPE and RMSE.

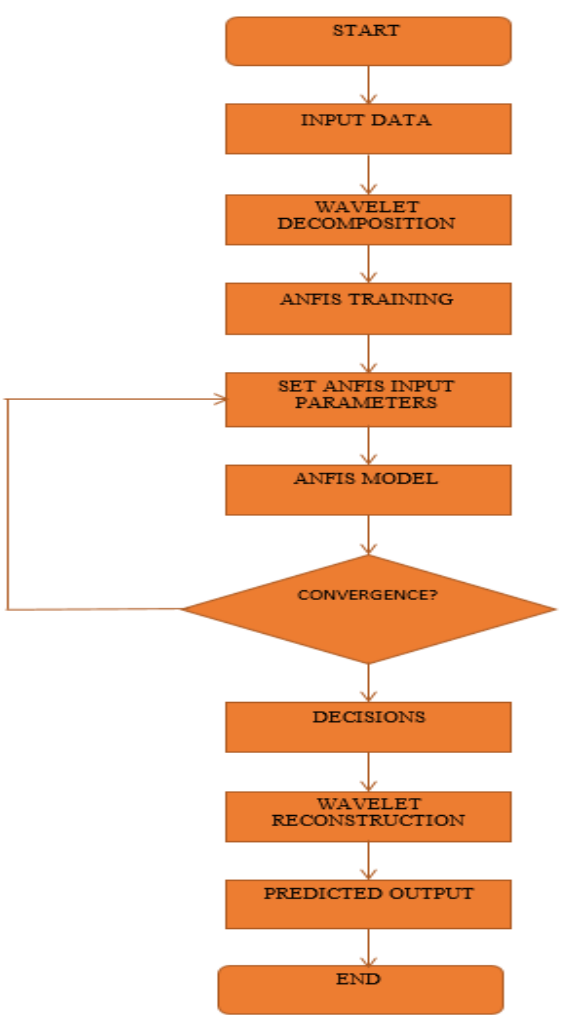

Figure 4. Flow chart of the developed WT-ANFIS model

\section{RESULTS AND ANALYSIS}

\subsection{Model Analysis}

In this study, Horizontal solar radiation is predicted using WT-ANFIS approach. Four inputs and one output parameters were used to develop and analyse the WT-ANFIS model. The predicted output and target of the WT-ANFIS solar radiation at the training phase are presented in Figure 5(a) while that of the testing phase are presented in Figure 5(b). The two figures shows a clear correlation between the target and the predicted output of the developed model both at the training and testing phase, the presented graphs clearly show perfect agreement between the predicted output and the target.

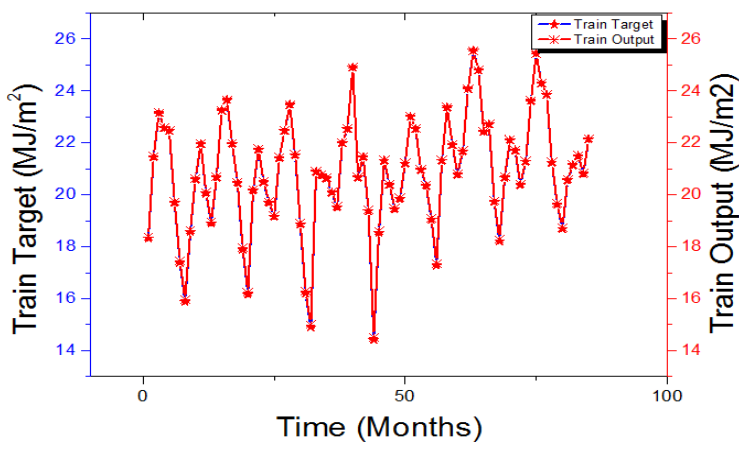

Figure 5(a). Training phase (actual and predicted)

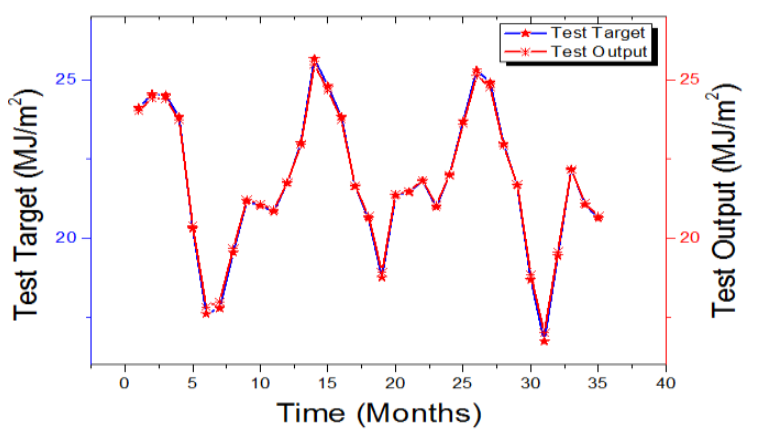

Figure 5(b). Testing phase (actual and predicted) 
Figure 6(a) presents the scatter plot at the training phase, $\mathrm{R}^{2}$ is very high at the training phase which is near +1 , and this proves a good model performance. Also, Figure 6(b) presents the scatter plot at the testing phase, $R^{2}$ is still high at the testing phase but lower than that of the training phase, it still proves good correlation because it approaches +1 .

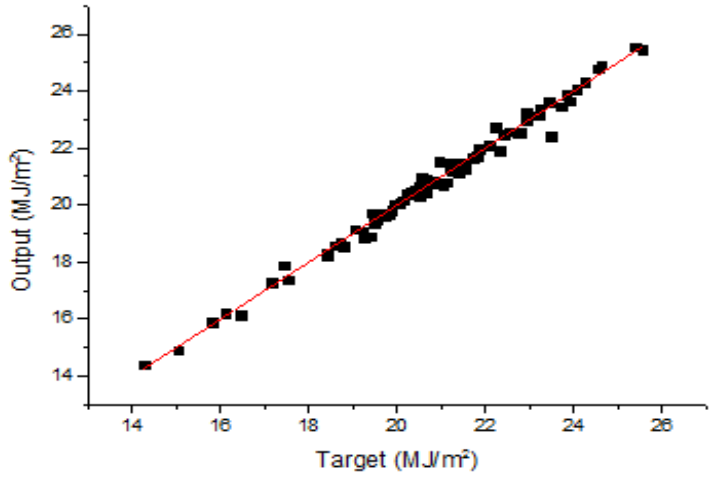

Figure 6(a). Scatter plot of the output against target (training phase)

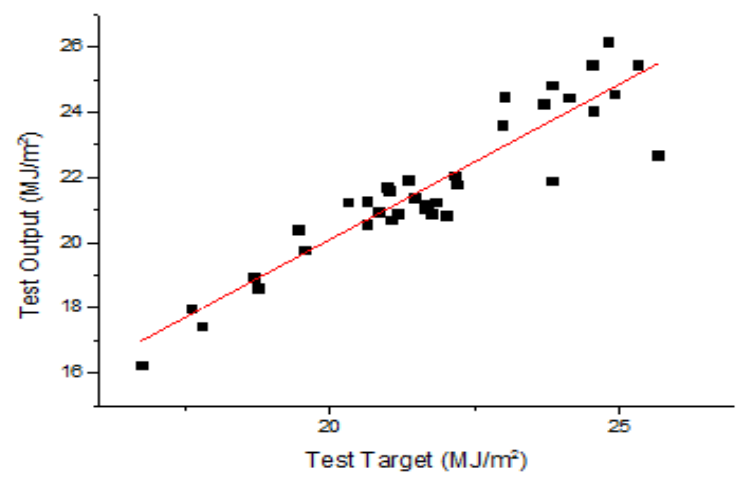

Figure 6(b). Scatter plot of the output against target (testing phase)

The statistical evaluators used to access the precision of the developed ANFIS-WT model are MAPE, RMSE and $\mathrm{R}^{2}$, Table 1 presents the results of the statistical evaluators at the training and testing phases. Lower values of RMSE and MAPE signifies good relationship, the ultimate value is 0 . The ideal value of $R^{2}$ ranges between 0 and 1 , for the value of $\mathrm{R}^{2}$ near 1 , it signifies a good linear relation and if it nears 0 it signifies nonlinear relation.

Table 1. WT-ANFIS model statistical evaluation

\begin{tabular}{llll}
\hline Data & RMSE & MAPE & $R^{2}$ \\
\hline Training & 0.23712 & 0.82161 & 0.9887 \\
Testing & 0.86759 & 1.5026 & 0.8584 \\
\hline
\end{tabular}

Table 2. Comparison with other models

\begin{tabular}{lllll}
\hline \multicolumn{1}{c}{ Reference } & Model & Case Study & \multicolumn{1}{c}{$\boldsymbol{R}^{\mathbf{2}}$} & RMSE \\
\hline Olatomiwa et al [5] & ANFIS & Nigeria & 0.8544 & 1.0854 \\
Remedani et al [17] & SVR-Poly & Iran & 0.8100 & 3.2000 \\
Olatomiwa et al [11] & SVR-Poly & Nigeria & 0.7703 & 1.3639 \\
Olatomiwa et al [12] & SVM-FFA & Nigeria & 0.8024 & 0.6988 \\
Remedani et al [17] & ANFIS & Iran & 0.808 & 3.8000 \\
Sajid and Ali [15] & ANFIS & Abu Dhabi & 0.860 & - \\
Remedani et al [17] & ANN & Iran & 0.7992 & 3.700 \\
Present study & WT-ANFIS & Nigeria & 0.9887 & 0.8216 \\
\hline
\end{tabular}

\subsection{Model validation}

The WT-ANFIS model validation was done by comparing the model results with existing literatures [5], [11], [12], [15] and [17]. The statistical evaluators used to compare the accuracy is $\mathrm{R}^{2}$ and RMSE, Table 2 presents the comparison between the results from different models and the WT- ANFIS result. From the table it clearly indicates that the developed WT-ANFIS model provides more precise prediction than the existing models based on $\mathrm{R}^{2}$ and RMSE values obtained.

\section{CONCLUSION}

In this study, WT-ANFIS was utilized for horizontal solar radiation prediction in Nigeria. WT is used to clean the data before the prediction exercise. Long term meteorological data comprising of monthly mean relative humidity, sunshine hours, maximum temperature, solar radiation and minimum temperature of the period (2002-2012) were used to train and test the model. The meteorological data selected for this 
research have strong linear relation with horizontal solar radiation and are readily available at the meteorological station in Nigeria. The developed WT-ANFIS model proves to be good model for horizontal solar radiation prediction. The statistical values of the MAPE, RMSE and $\mathrm{R}^{2}$ obtained are $0.23712,0.82161$ and 0.9887 respectively. Based on the values of $\mathrm{R}^{2}$ used for comparison between the developed model and the validated models, the WT-ANFIS show better accuracy and performance. Also, by adding more meteorological data more prediction accuracy is attained. With the obtained results, it indicates that the addition of WT for data decomposition and reconstruction improves the ANFIS model accuracy for horizontal solar radiation prediction. More meteorological data will be considered in future study and new models will be developed using new soft computing techniques.

\section{ACKNOWLEDGEMENT}

The authors are thankful to Universiti Teknologi Malaysia for providing International Doctoral Fellowship (IDF) award to the student and their continuous support.

\section{REFERENCES}

[1] K. Mohammadi, S. Shamshirband, A. S. Danesh, M. S. Abdullah, and M. Zamani, "Temperature-based estimation of global solar radiation using soft computing methodologies," Theoretical and Applied Climatology, Vol 121, pp. $1-12,2015$.

[2] A. Trabea and M. M. Shaltout, "Correlation of global solar radiation with meteorological parameters over Egypt," Renewable Energy, vol. 21, pp. 297-308, 2000.

[3] K. Chiteka and C. Enweremadu, "Prediction of global horizontal solar irradiance in Zimbabwe using artificial neural networks," Journal of Cleaner Production, vol. 135, pp. 701-711, 2016.

[4] M. Alam, S. K. Saha, M. Chowdhury, M. Saifuzzaman, and M. Rahman, "Simulation of solar radiation system," American Journal of Applied Sciences, vol. 2, pp. 751-758, 2005.

[5] L. Olatomiwa, S. Mekhilef, S. Shamshirband, and D. Petkovic, "Adaptive neuro-fuzzy approach for solar radiation prediction in Nigeria," Renewable \& Sustainable Energy Reviews, vol. 51, pp. 1784-1791, Nov 2015.

[6] F. Besharat, A. A. Dehghan, and A. R. Faghih, "Empirical models for estimating global solar radiation: A review and case study," Renewable and Sustainable Energy Reviews, vol. 21, pp. 798-821, 2013.

[7] J. L. Chen and G. S. Li, "Estimation of monthly average daily solar radiation from measured meteorological data in Yangtze River Basin in China," International Journal of Climatology, vol. 33, pp. 487-498, 2013.

[8] J. Wu, C. K. Chan, Y. Zhang, B. Y. Xiong, and Q. H. Zhang, "Prediction of solar radiation with genetic approach combing multi-model framework," Renewable Energy, vol. 66, pp. 132-139, 2014.

[9] M. Trnka, Z. Žalud, J. Eitzinger, and M. Dubrovský, "Global solar radiation in Central European lowlands estimated by various empirical formulae," Agricultural and Forest Meteorology, vol. 131, pp. 54-76, 2005.

[10] G. H. Hargreaves and Z. A. Samani, "Estimating potential evapotranspiration," Journal of the Irrigation and Drainage Division, vol. 108, pp. 225-230, 1982.

[11] L. Olatomiwa, S. Mekhilef, S. Shamsirband and D. Petkovic, Potential of Support Vector Regression for Solar Radiation Prediction in Nigeria, "Natural Hazard, vol. 77, pp 1055-1068, 2015.

[12] L. Olatomiwa, S. Mekhilef, S. Shamsirband, K. Mohammadi, D. Petkovic and C. Sudheer. A Support Vector Machine Firefly Algorithm-based Model for Solar Radiation Prediction, "Solar Energy, vol. 115, pp. 632-644, 2015

[13] S. Salisu, A. Abubakar, B. Sadiq, A. Abdu, and A. Umar, "FORCASTING SOLAR RADIATION INTENSITY USING ANN AND ANFIS (A COMPARATIVE STUDY AND PERFORMANCE ANALYSIS), International Engineering Conference, FUT Minna" pp. 567-571, 2015.

[14] K. Mohammadi, S. Shamshirband, C. W. Tong, K. A. Alam, and D. Petković, "Potential of adaptive neuro-fuzzy system for prediction of daily global solar radiation by day of the year," Energy Conversion and Management, vol. 93, pp. 406-413, 2015.

[15] S. Hussain and A. Al Alili, "Soft computing approach for solar radiation prediction over Abu Dhabi, UAE: A comparative analysis," IEEE International Conference on in Smart Energy Grid Engineering (SEGE), pp. 1-6, 2015.

[16] M. Sedighi, M. Ghasemi, M. Mohammadi, and S. H. Hassan, "A novel application of a neuro-fuzzy computational technique in modeling of thermal cracking of heavy feedstock to light olefin," RSC Advances, vol. 4, pp. 2839028399, 2014.

[17] Z. Ramedani, M. Omid, A. Keyhani, S. Shamshirband, and B. Khoshnevisan, "Potential of radial basis function based support vector regression for global solar radiation prediction," Renewable and Sustainable Energy Reviews, vol. 39, pp. 1005-1011, 2014.

[18] NIMET, "Nigerian Meteorological Agency, Kano, Kano State, Nigeria," 2016.

[19] NASA, "Surface meteorology and Solar Energy, https://eosweb.larc.nasa.gov/sse/," 2016.

[20] J. Rafiee, P. Tse, A. Harifi, and M. Sadeghi, "A novel technique for selecting mother wavelet function using an intelligent fault diagnosis system," Expert Systems with Applications, vol. 36, pp. 4862-4875, 2009. 
[21] A. Pretto, E. Menegatti, Y. Jitsukawa, R. Ueda, and T. Arai, "Image similarity based on Discrete Wavelet Transform for robots with low-computational resources," Robotics and Autonomous Systems, vol. 58, pp. 879-888, 2010.

[22] Z. Bashir and M. El-Hawary, "Applying wavelets to short-term load forecasting using PSO-based neural networks," IEEE Transactions on Power Systems, vol. 24, pp. 20-27, 2009.

[23] S. Li, P. Wang, and L. Goel, "A novel wavelet-based ensemble method for short-term load forecasting with hybrid neural networks and feature selection," IEEE Transactions on power systems, vol. 31, pp. 1788-1798, 2016.

[24] J.-S. Jang, "ANFIS: adaptive-network-based fuzzy inference system," IEEE transactions on systems, man, and cybernetics, vol. 23, pp. 665-685, 1993.

[25] M. Mustapha, M.W. Mustafa, S. Khalid, I. Abubakar, and A. M. Abdilahi, "Correlation and Wavelet-based ShortTerm Load Forecasting using Anfis," Indian Journal of Science and Technology, vol. 9, 2016.

[26] F. Kocabaş and Ş. Ülker, "Estimation of critical submergence for an intake in a stratified fluid media by neurofuzzy approach," Environmental Fluid Mechanics, vol. 6, pp. 489-500, 2006.

[27] G. Landeras, J. J. López, O. Kisi, and J. Shiri, "Comparison of Gene Expression Programming with neuro-fuzzy and neural network computing techniques in estimating daily incoming solar radiation in the Basque Country (Northern Spain)," Energy Conversion and Management, vol. 62, pp. 1-13, 2012.

[28] J.-S. R. Jang, C.-T. Sun, and E. Mizutani, "Neuro-fuzzy and soft computing; a computational approach to learning and machine intelligence," 1997.

[29] T. Nguyen and Y. Liao, "Short-Term Load Forecasting Based on Adaptive Neuro-Fuzzy Inference System," Journal of computers, vol. 6, pp. 2267-2271, 2011.

[30] M. Sugeno and G. Kang, "Structure identification of fuzzy model," Fuzzy sets and systems, vol. 28, pp. 15-33, 1988. 